Journal of

\section{Applied Crystallography}

ISSN 0021-8898

Received 16 August 2006

Accepted 22 January 2007

\title{
Collapse of the hydration shell of a protein prior to thermal unfolding
}

\author{
Masaharu Koizumi, ${ }^{a}$ Harutaka Hirai, ${ }^{a}$ Teruaki Onai, ${ }^{a}$ Katsuaki Inoue $^{\mathrm{b}}$ and \\ Mitsuhiro Hirai ${ }^{\text {a* }}$
}

aDepartment of Physics, Gunma University, Maebashi 371-8510, Japan, and ${ }^{\mathbf{b}}$ Japan Synchrotron Radiation Research Institute, Sayo Hyogo 679-5198, Japan. Correspondence e-mail: mhirai@fs.aramaki.gunma-u.ac.jp

Based on high statistical quality wide-angle X-ray scattering data for the unfolding-refolding process of hen egg-white lysozyme (HEWL), we have analysed the change of the hydration shell as a function temperature using the program CRYSOL. The present results suggest that the decrease of the hydration-shell density starts from a lower temperature than the transition temperature of the collapse of the tertiary structure of HEWL. Although the use of CRYSOL for scattering data for proteins before the transition has an apparent limitation, the collapse of the hydration shell prior to the unfolding of HEWL agrees with a slight tendency of the radius of gyration to decrease during the thermal unfolding process.

(C) 2007 International Union of Crystallography Printed in Singapore - all rights reserved

\section{Introduction}

Hydration of biological macromolecules is assumed to have an important role in their structural stability and functions (Israelachvili \& Wennerström, 1996). As suggested by inelastic neutron scattering experiments, the functional properties and dynamics of proteins in solution are coupled with the dynamic behaviour of the surrounding water molecules (Gabel et al., 2002). Studies of proteins using X-ray and neutron solution scattering techniques indicate that the density of water around the protein surface is much higher than that of bulk water (Svergun et al., 1998). They showed the existence of a hydration shell whose average density is around $10 \%$ larger than that of the bulk water. Molecular dynamics simulation studies also suggested that the variation in the first hydration shell density is subject to the electrostatic properties of the protein surface and local surface topography (Makarov et al., 1998, 2000; Dastidar \& Mukhopadhyay, 2003). However, the properties of the hydrated water shells are still the subject of many experimental and theoretical investigations.

On the other hand, the mechanism by which proteins fold into their native structures is one of essential problems in biology and is still under extensive study (Pain, 2000; Oliveberg \& Wolynes, 2006). To clarify the mechanism of protein folding, various methods have been used to observe detailed features of the folding-unfolding processes of proteins (Dobson, 2004). Hydration of proteins is assumed to greatly contribute not only to their structural stability but also their functions. By using synchrotron radiation (SR) wide-angle X-ray scattering and differential scanning calorimetry (DSC), we have been studying the thermal unfolding-refolding process of hen egg-white lysozyme (HEWL) at various $\mathrm{pH}$ values under stepwise heating and cooling conditions (Hirai et al., 1998, 1999; Arai \& Hirai, 1999). Recently we demonstrated that the wide-angle X-ray scattering (WAXS) method using high-intensity X-rays from a third-generation synchrotron-radiation source enables us to observe directly the whole hierarchical structure of proteins including quaternary, tertiary, domain and secondary structures in solution (Hirai et al., 2002), and we showed that the structures of proteins in solutions can be compared with crystallographic structures by using a program such as
CRYSOL (Svergun et al., 1995). By using SR-WAXS we successfully analysed the details of the reversible unfolding-refolding process of HEWL covering all the hierarchical structures from tertiary to secondary structure (over the real-space distance from $\sim 2.5$ to $\sim 125 \AA$ ), and showed that the $\mathrm{pH}$ dependence of the thermal structural transition of HEWL is characterized by its hierarchical structures and the cooperativity between them (Hirai et al., 2004). In that report we presented a new hierarchical map of unfoldingrefolding transitions by determining the molar ratios of the nativestructure protein for each hierarchical level at an intermediate transition state, which would be comparable to the fraction of native contacts used in a 'folding funnel' representation (Dill \& Chan, 1997; Dinner et al., 2000). Thus, the SR-WAXS method is widely applicable to the studies of various proteins (Fischetti et al., 2003, 2004) and enables discussion of the relation between the structural transitions and intramolecular structure of proteins (Koizumi et al., 2006).

In the present report we have evaluated the change of the hydration shell during the thermal unfolding-refolding transition by using the CRYSOL program to analyse the WAXS data of HEWL at the transition. We have found that the initial stage of HEWL unfolding accompanies a collapse of the hydration shell. In the present analyses we have used the WAXS data reported previously (Hirai et al., 2004). We will also discuss the feasibility and limitation of the present analysis.

\section{Materials and method}

\subsection{Sample preparation}

Three-times crystallized HEWL from Sigma Chemical Co. was used for WAXS measurements. HEWL was dissolved in water whose $\mathrm{pH}$ was adjusted by adding $\mathrm{HCl}$ before the dissolution. In many cases of protein-folding studies, an aggregation of proteins occurs in the unfolding and/or refolding process. As reported previously (Hirai et al., 1998, 1999), the present solvent conditions can avoid such aggregation due to the repulsive interparticle interaction between proteins through the Coulomb potential. Final $\mathrm{pH}$ values of the 
protein solutions were determined by using a digital HM-60V pH meter from TOA Electronics Ltd. The concentrations of the samples were $0.05 \mathrm{~g} \mathrm{ml}^{-1}$ for the thermal unfolding-refolding measurements and $0.01 \mathrm{~g} \mathrm{ml}^{-1}$ for the $\mathrm{pH}$ dependence measurements.

\subsection{Small-angle and wide-angle $X$-ray scattering measurements}

The spectrometer used for SR-WAXS experiments was an X-ray scattering spectrometer installed at BL-40B2 of the $8 \mathrm{GeV}$ synchrotron-radiation source of the Japan Synchrotron Radiation Research Institute (JASRI), Harima, Japan. Details of the spectrometer have been described elsewhere (Miura et al., 2000). The sample-todetector distance and the X-ray wavelength used were $41 \mathrm{~cm}$ and $0.729 \AA$, respectively. The scattering intensity was recorded by an RAXIS IV imaging plate system from Rigaku. The intensities of the incident and transmitted X-rays were monitored by a pair of ionization chambers. A sample cell composed of a pair of thin quartz windows with $1 \mathrm{~mm}$ path length was used. The exposure time to the $\mathrm{X}$-rays was $30 \mathrm{~s}$ for each measurement. The $q$ values of the circularly averaged scattering intensity $I(q)$ were calibrated using the diffraction peaks of silver behenate (Huang et al., 1993), where $q=(4 \pi /$ $\lambda) \sin \theta, \lambda$ is the wavelength and $2 \theta$ is the scattering angle. The beam size at the sample position was $\sim 0.1 \mathrm{~mm}^{2}$. Other details of the WAXS measurements are given in the previous reports (Hirai et al., 2002, 2004).

\subsection{Scattering data treatment and model analysis}

To obtain the net scattering from the proteins the following equation was used (Hirai et al., 2002, 2004).

$$
\begin{aligned}
I(q)= & {\left[I_{\text {sol }}(q) /\left(B_{\text {sol }} T_{\text {sol }}\right)-I_{\text {cell }}(q) /\left(B_{\text {cell }} T_{\text {cell }}\right)\right] / P_{\text {sol }} } \\
& \left.-\left(1-c v_{\alpha}\right)\left[I_{\text {solv }}(q) /\left(B_{\text {solv }} T_{\text {solv }}\right)-I_{\text {cell }}(q) / B_{\text {cell }} T_{\text {cell }}\right)\right] / P_{\text {solv }}
\end{aligned}
$$

where $I_{\text {sol }}(q), I_{\text {solv }}(q), I_{\text {cell }}(q), B_{\text {sol }}, B_{\text {solv }}, B_{\text {cell }}, T_{\text {sol }}, T_{\text {solv }}$ and $T_{\text {cell }}$ are the observed scattering intensities, the incident beam intensities and the transmissions of the solution and the solvent at each temperature and the sample cell, respectively. $P_{\text {sol }}$ and $P_{\text {solv }}$ are the watercorrelation peak intensities of the solution and the solvent; $c$ and $v_{\alpha}$ are the concentration of the protein molecule and its partial specific volume, respectively. The $v_{\alpha}$ value used was $0.712 \mathrm{ml} \mathrm{g}^{-1}$ for HEWL (Gekko \& Hasegawa, 1986). In the present modelling analysis we used the CRYSOL program. This program is well known for fitting experimental scattering curves of proteins in solutions based on their crystallographic coordinates by considering the hydration shell (Svergun et al., 1995, 1998). WAXS data in the $q$ range from 0.09 to
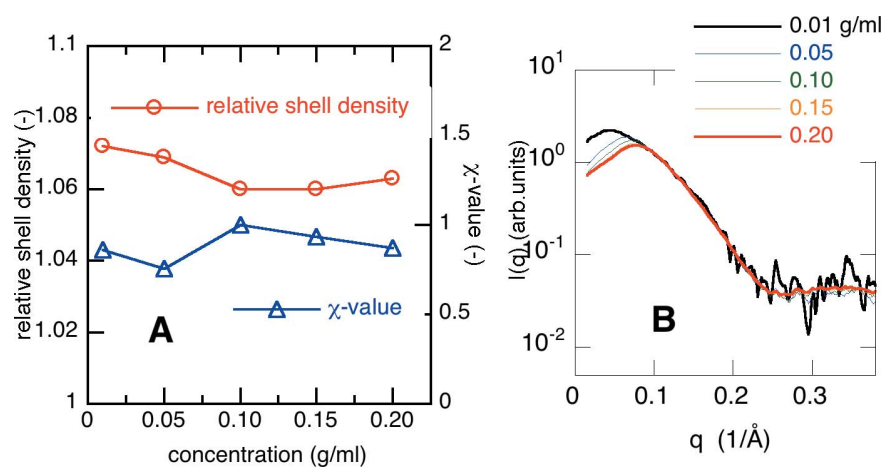

Figure 1

(A) Concentration dependence of the HSD and $\chi$ values. (B) Scattering data from the HEWL solutions used for HSD estimation, with concentrations ranging from 0.01 to $0.2 \mathrm{~g} \mathrm{ml}^{-1}$ in $50 \mathrm{~m} M$ Hepes at $\mathrm{pH} 7$ at $293 \mathrm{~K}$ (reproduced from Hirai et al., 1998).
$1.8 \AA^{-1}$ were used for fitting based on the crystallographic data of HEWL [6LYZ from Diamond (1974)] using the CRYSOL program using 50 spherical harmonics and default parameters. Therefore, the only fitting parameter was the hydration shell density (HSD).

\section{Results and discussion}

\subsection{Concentration and $\mathrm{pH}$ dependence of the hydration shell} density

As we used relatively high concentration protein solutions for obtaining high statistical quality WAXS data, we have checked the effect of the protein concentration on the HSD value by using CRYSOL with previous data for HEWL (Hirai et al., 1998). Fig. 1A shows the concentration dependence of the HSD and $\chi$ values obtained, and Fig. 1B shows the scattering data from 0.01 to $0.2 \mathrm{~g} \mathrm{ml}^{-1} \mathrm{HEWL}$ in $50 \mathrm{~m} M$ Hepes at $\mathrm{pH} 7$ at $293 \mathrm{~K}$ (Hirai et al., 1998). The $\chi$ value is defined by

$$
\chi^{2}=\frac{1}{N-1} \sum_{j=1}^{N}\left[\frac{I_{\text {theo }}\left(q_{j}\right)-I_{\text {exp }}\left(q_{j}\right)}{\sigma\left(q_{j}\right)}\right],
$$

where $N$ is the number of experimental data points, $I_{\text {theo }}(q)$ and $I_{\exp }(q)$ are the theoretical and experimental intensities, and $\sigma(q)$ is the standard deviation of $I_{\exp }(q)$ (Svergun et al., 1998).

In Fig. 1A the estimated HSD values below a concentration of $0.05 \mathrm{~g} \mathrm{ml}^{-1}$ are comparable to the reported value of 1.07 (Svergun et $a l ., 1998)$ in spite of the use of high- $q$ data $\left(q>0.09 \AA^{-1}\right)$, where the average scattering density of water was taken as 1.0. Fig. $2 \mathrm{~A}$ shows the $\mathrm{pH}$ dependence of the HSD and radius of gyration $R_{\mathrm{g}}$ of $0.01 \mathrm{~g} \mathrm{ml}^{-1}$ HEWL, and Fig. 2B shows the WAXS data used for the calculation (Hirai et al., 2004). As shown in Fig. 2A, the values of the HSD and $R_{\mathrm{g}}$ are mostly independent of $\mathrm{pH}$ in the wide $\mathrm{pH}$ range from 1.9 to 6.3 , and give reasonable values of $\sim 1.07$ and $15.4 \pm 0.2 \AA$, respectively. Figs. $1 \mathrm{~A}$ and $2 \mathrm{~A}$ suggest the scattering data above $q=$ $0.09 \AA^{-1}$ reflect the contribution of the hydration shell well and are enough for estimating the hydration shell density and $R_{\mathrm{g}}$ using CRYSOL in the case of HEWL. This situation can be understood from Fig. 3. Fig. 3 is the theoretical WAXS curve and $R_{\mathrm{g}}$ value of HEWL depending on the relative value of the average excess scattering density, the so-called contrast of the hydration shell defined by (HSD - 1.0), which was obtained by CRYSOL. The increase of the HSD from 1.0 to 1.06 increases $R_{\mathrm{g}}$ from 14.01 to $15.30 \AA$ and changes the scattering profile below $\sim 0.8 \AA^{-1}$. The experimental $R_{\mathrm{g}}$ value of $15.4 \pm 0.2 \AA$ at $298 \mathrm{~K}$ at $\mathrm{pH} 4.6$ corresponds to the theoretical $R_{\mathrm{g}}$ value at the relative shell contrast of 0.068 , which is in agreement with

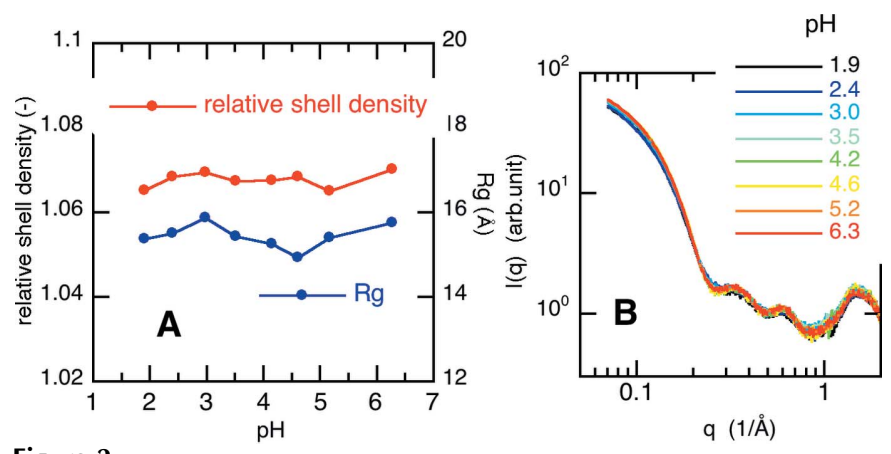

Figure 2

(A) $\mathrm{pH}$ dependence of the HSD and radius of gyration $R_{\mathrm{g}}$ of $0.01 \mathrm{~g} \mathrm{ml}^{-1}$ solutions of HEWL at $298 \mathrm{~K}$. (B) WAXS data used for the calculations (reproduced from Hirai et al., 2004). 

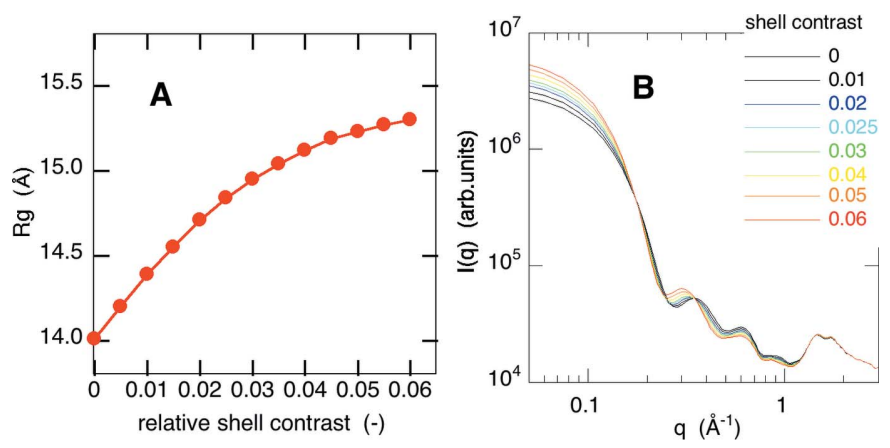

Figure 3

(A) Radius of gyration of HEWL as a function of the contrast of the hydration shell. (B) Shell-contrast dependence of the theoretical WAXS curves. (A) and (B) were obtained from $C R Y S O L$ using data for $6 \mathrm{LYZ}$. The contrast was varied from 0 to 0.06 .

the reported value (Svergun et al., 1998). Thus, WAXS curves above $q$ $=0.09 \AA^{-1}$ are assumed to reflect the change of the HSD well in spite of the use of relatively high $q$ values. It should be mentioned that the change of the shell contrast does not affect the theoretical WAXS curves above $q=\sim 1 \AA^{-1}$.

On the other hand, in the case of the presence of interparticle interaction between solute particles the $R_{\mathrm{g}}$ value evaluated using a Guinier plot $\left[\ln I(q)\right.$ versus $\left.q^{2}\right]$ is known to be sensitive to the $q$ range used for calculation. Fig. 4 shows the concentration dependence of $R_{\mathrm{g}}$ values estimated by a Guinier plot using different $q$ ranges. Fig. 4 suggests that the effect of repulsive interparticle interaction on the scattering curve appearing as a broad peak below $q=\sim 0.065 \AA^{-1}$ for $0.05 \mathrm{~g} \mathrm{ml}^{-1}$ can be neglected when we use the scattering data above $q$ $=0.09 \AA^{-1}$, which also confirms that the use of a relatively high $q$ value is reasonable to avoid the effect of interparticle interaction on scattering data analysis for high concentration samples.

\subsection{Temperature dependence of experimental WAXS curves compared with fitted theoretical curves}

Fig. 5 shows a part of the experimental WAXS curves of $0.05 \mathrm{~g} \mathrm{ml}^{-1} \mathrm{HEWL}$ on heating from 285 to $352 \mathrm{~K}$ (Hirai et al., 2004)

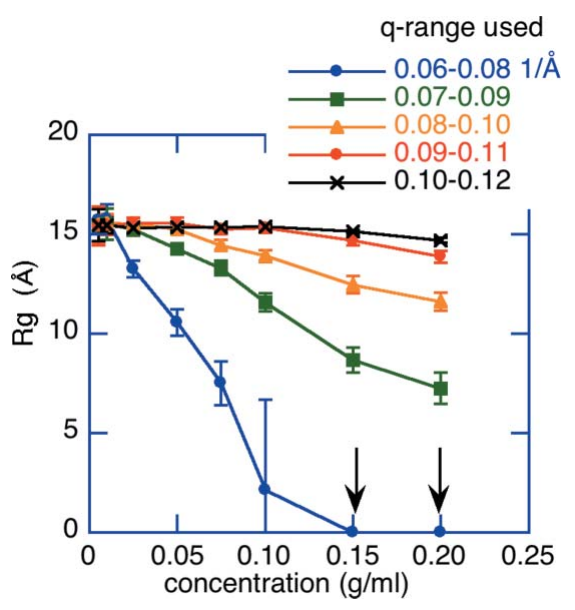

Figure 4

Radius of gyration, $R_{\mathrm{g}}$, of HEWL (pH 7.0, $298 \mathrm{~K}$ ) as a function of concentration. The $R_{\mathrm{g}}$ values were estimated by applying a Guinier plot to the scattering data for the different $q$ ranges shown in the figure. The arrows indicate the $R_{\mathrm{g}}$ values that are indeterminable due to the presence of a broad interparticle correlation peak. The data used are from Arai \& Hirai (1999).

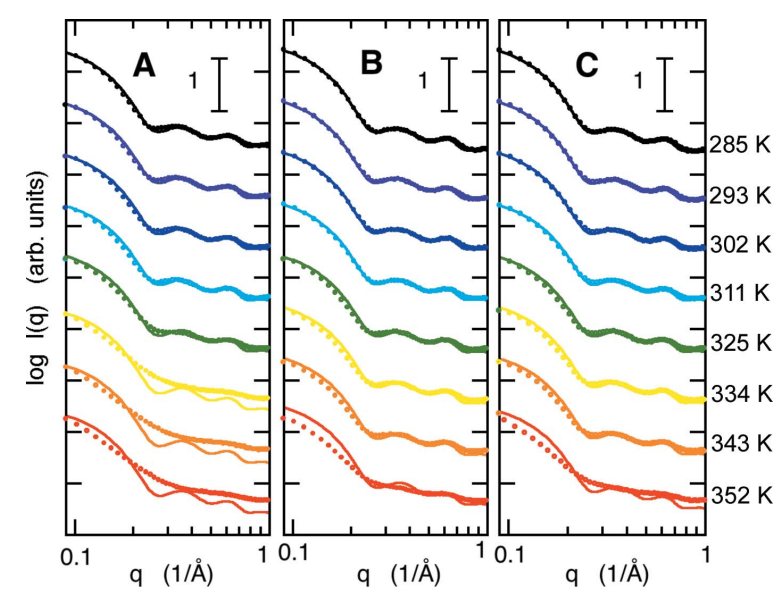

Figure 5

Comparison of the experimental WAXS curves of $0.05 \mathrm{~g} \mathrm{ml}^{-1} \mathrm{HEWL}$ at different temperatures with the fitted theoretical WAXS curves obtained from CRYSOL. (A) at $\mathrm{pH} 2.2$; (B) at $\mathrm{pH} 3.6$; (C) at $\mathrm{pH} 4.5$. The dots and thick lines correspond to the experimental data and the fitted theoretical WAXS curves, respectively. The experimental data are reproduced from Hirai et al. (2004).

compared with the fitted theoretical curves obtained using CRYSOL, where $\mathrm{A}, \mathrm{B}$ and $\mathrm{C}$ correspond to $\mathrm{pH} 2.2,3.6$ and 4.5, respectively. In the temperature range $285-318 \mathrm{~K}$ the experimental and theoretical WAXS curves at $\mathrm{pH} 3.6$ and 4.5 show good agreement over the whole $q$ range. On the other hand, the theoretical WAXS curve at $\mathrm{pH} 2.2$ shows a slight deviation from the experimental one even at $285 \mathrm{~K}$. At
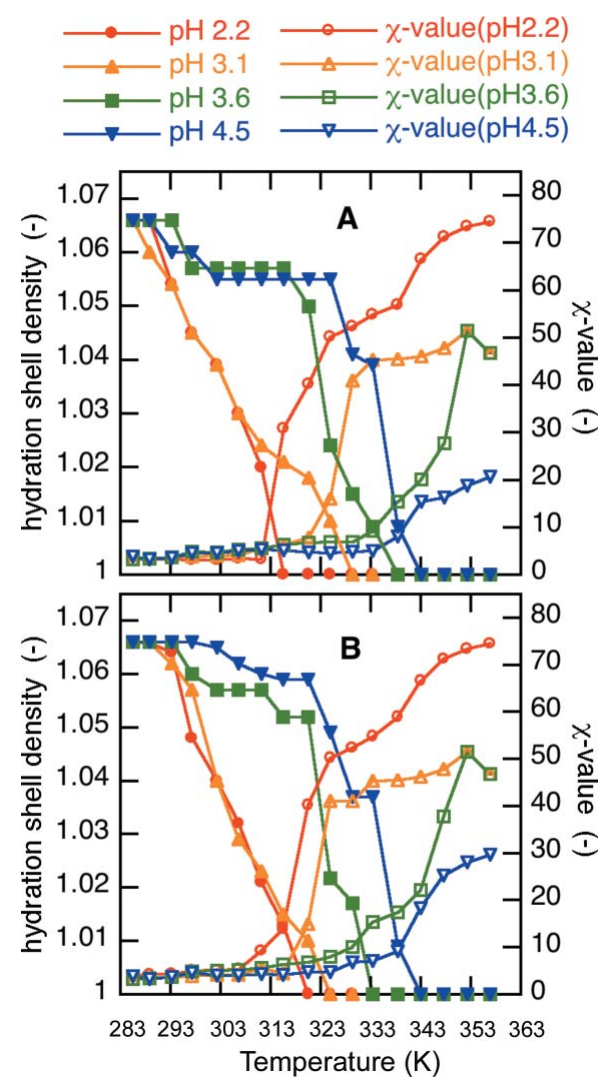

Figure 6

Temperature dependence of the relative value of the hydration shell density and the $\chi$ value obtained by CRYSOL, where (A) and (B) correspond to the heating (unfolding) and cooling (refolding) processes, respectively. 
all $\mathrm{pH}$ values the deviation becomes evident above the transition temperature as shown in $\S 3.3$. Thus, Fig. 5 indicates that the fitting procedure using CRYSOL would be applicable to the WAXS data especially at $\mathrm{pH} 3.6$ and 4.5 below $\sim 318 \mathrm{~K}$, suggesting that the relative value of the HSD estimated by $C R Y S O L$ would be reasonable below this temperature.

\subsection{Temperature dependence of $\chi$ and the hydration shell density of HEWL at unfolding and refolding}

Based on the above consideration, we have evaluated the change of the relative value of the HSD of HEWL in the thermal unfolding and refolding processes by using CRYSOL with all WAXS data reported previously (Hirai et al., 2004). Figs. 6A and 6B show the temperature dependence of the relative value of the HSD at different $\mathrm{pH}$ values and the $\chi$ value obtained by CRYSOL, where $\mathrm{A}$ and $\mathrm{B}$ correspond to the heating (unfolding) and cooling (refolding) processes, respectively. As reported previously, the onset $\left(T_{\text {on }}\right)$ and midpoint $\left(T_{\mathrm{m}}\right)$ temperatures of the HEWL thermal transition observed by DSC agree well with the structural transition temperatures observed from the change of $R_{\mathrm{g}}$ (Hirai et al., 1999), and the thermal unfolding and refolding processes of HEWL are highly reversible (Arai \& Hirai, 1999; Hirai et al., 2004). The $T_{\text {on }}$ and $T_{\mathrm{m}}$ of $R_{\mathrm{g}}$ observed were $T_{\text {on }}=\sim 339 \mathrm{~K}$ and $T_{\mathrm{m}}=\sim 349 \mathrm{~K}$ for $\mathrm{pH} 4.5, T_{\text {on }}=$ $\sim 335 \mathrm{~K}$ and $T_{\mathrm{m}}=\sim 346 \mathrm{~K}$ for $\mathrm{pH} 3.6, T_{\mathrm{on}}=\sim 332 \mathrm{~K}$ and $T_{\mathrm{m}}=\sim 344 \mathrm{~K}$ for $\mathrm{pH} 3.1$, and $T_{\mathrm{on}}=\sim 318 \mathrm{~K}$ and $T_{\mathrm{m}}=\sim 326 \mathrm{~K}$ for $\mathrm{pH} 2.2$ (Hirai et al., 2004). As shown in Figs. 6A and 6B, the change of the HSD is mostly reversible both in the thermal unfolding and refolding processes. The significant increase of the $\chi$ value starts from around $T_{\text {on }}$ at every $\mathrm{pH}$ value, especially at $\mathrm{pH} 4.5$ and 3.6. It is clear that the relative values of the HSD above the temperature at which the $\chi$ value shows a significant increase are meaningless. On the other hand, even below $T_{\text {on }}$, where the change of the $\chi$ value is relatively small, the relative value of the HSD estimated shows a decreasing tendency for all $\mathrm{pH}$ values. This tendency agrees with the finding of a slight decrease of $R_{\mathrm{g}}$ below $T_{\text {on }}$ reported previously (Hirai et al., 1998, 1999). Thus, Fig. 6 suggests that before the main transition of the tertiary structure of HEWL a collapse or change of the hydration shell begins. Alternatively, the elevation of temperature affects at first the change of the hydration shell through the enhancement of the thermal fluctuation of the protein, destabilizes the native folded structure, and finally induces the collapse of the tertiary structure with an accompanying significant increase of $R_{\mathrm{g}}$ and a large change of Gibbs free energy (Hirai et al., 1998, 1999; Arai \& Hirai, 1999).

The present interpretation would be reasonable as follows. The hydration shell of a protein results from different types of hydration, namely, electrostatic hydration by dissociation amino acids, hydrophobic hydration by non-polar amino acids, and hydration by hydrogen bonds by polar amino acids. Even below $T_{\text {on }}$, the thermal expansion of the tertiary structure due to the enhancement in the intramolecular fluctuation accompanies further exposure of the hydrophobic core within the protein, which would increase the hydrophobic hydration that decreases the HSD. Thus, the thermal expansion and the collapse of the hydrophilic hydration shell would contribute to the change of $R_{\mathrm{g}}$ in opposite ways. Alternatively, in the case of HEWL the thermal expansion and the collapse of the hydrophilic hydration shell would begin simultaneously; however, the latter effect on $R_{\mathrm{g}}$ would be dominant below $T_{\mathrm{on}}$ as found previously in the slight decrease of $R_{\mathrm{g}}$ (Hirai et al., 1998, 1999). The change of the HSD suggested in the present study might be confirmed by using other experimental and theoretical methods such as nuclear magnetic resonance and molecular dynamics simulation.

The present results suggest that the use of the program CRYSOL would be applicable not only to the comparison of protein structures in solutions with those in crystal states but also to the estimation of the changes of HSDs for small proteins for which the tertiary structures are mostly native-like. We should mention that the present analysis is not applicable to the case of the thermal unfolding of $\alpha$ lactalbumin $(\alpha$-LA), a homologous protein of HEWL, since the observed WAXS profile of $\alpha$-LA in solution at room temperature is evidently different from the theoretical one in the crystal state calculated by CRYSOL (Koizumi et al., 2006). Thus, the reasonability of estimated HSDs must be judged both by the $\chi$ value obtained and by the agreement of the fitted theoretical WAXS curves with the experimental ones.

The WAXS experiments were done under the approval of the JASRI Program Advisory Committee (Proposal Nos. 2001B0455 and 2004B0082).

\section{References}

Arai, S. \& Hirai, M. (1999). Biophys. J. 76, 2192-2197.

Dastidar, S. G. \& Mukhopadhyay, C. (2003). Phys. Rev. E, 68, 21921-21930.

Diamond, R. (1974). J. Mol. Biol. 82, 371-391.

Dill, K. A. \& Chan, H. S. (1997). Nature Struct. Biol. 4, 10-19.

Dinner, A. R., Sali, A., Smith, L. J., Dobson, C. M. \& Karplus, M. (2000). Trends Biochem. Sci. 25, 331-339.

Dobson, C. M. (2004). Methods, 34, 4-14.

Fischetti, R. F., Rodi, D. J., Gore, D. B. \& Makowski, L. (2004). Chem. Biol. 11, 1431-1443.

Fischetti, R. F., Rodi, D. J., Mirza, A., Irving, T. C., Kondrashkina, E. \& Makowski, L. (2003). J. Synchrotron Rad. 10, 398-404.

Gabel, F., Bicout, D., Lehnert, U., Tehei, M., Weik, M. \& Zaccai, G. (2002). Q. Rev. Biophys. 35, 327-367.

Gekko, K. \& Hasegawa, Y. (1986). Biochemistry, 25, 6563-6571.

Hirai, M., Arai, S. \& Iwase, H. (1999). J. Phys. Chem. B, 103, 549-556.

Hirai, M., Arai, S., Iwase, H. \& Takizawa, T. (1998). J. Phys. Chem. B, 102, 1308-1313.

Hirai, M., Iwase, H., Hayakawa, T., Miura, K. \& Inoue, K. (2002). J. Synchrotron Rad. 9, 202-205.

Hirai, M., Koizumi, M., Hayakawa, T., Takahashi, H., Abe, S., Hirai, H., Miura, K. \& Inoue, K. (2004). Biochemistry, 43, 9036-9049.

Huang, T. C., Toraya, H., Blanton, T. N. \& Wu, Y. (1993). J. Appl. Cryst. 26, 180-184.

Israelachvili, J. \& Wennerström, H. (1996). Nature (London), 379, 219-225.

Koizumi, M., Hirai, M. \& Inoue, K. (2006). Bunseki Kagaku, 55, 411-418.

Makarov, V. A., Andrews, B. K. \& Pettitt, B. M. (1998). Biopolymers, 45, 469478.

Makarov, V. A., Andrews, B. K., Smith, P. E. \& Pettitt, B. M. (2000). Biophys. J. 79, 2966-2974.

Miura, K., Kawamoto, M., Inoue, K., Yamamoto, M., Kumasaka, T., Sugiura, M., Yamano, A. \& Moriyama, H. (2000). SPring-8 User Exp. Rep. 4, 168.

Oliveberg, M. \& Wolynes, P. G. (2006). Q. Rev. Biophys. 19, 1-44.

Pain, R. H. (2000). Editor. Mechanisms of protein folding. Oxford University Press.

Svergun, D. I., Barberato, C. \& Koch, M. H. J. (1995). J. Appl. Cryst. 28, 768773 .

Svergun, D. I., Richard, S., Koch, M. H. J., Sayers, Z., Kuprin, S. \& Zaccai, G. (1998). Proc. Natl Acad. Sci. USA, 95, 2267-2272. 